\title{
A Study on Diagnosis Support System for Application of Medical Information
}

\author{
Sung-In Kang ${ }^{1}$, Gwan-Hyung $\mathrm{Kim}^{2}$ and $\mathrm{Am}-\mathrm{Suk} \mathrm{Oh}^{3}$ \\ ${ }^{1}$ Dept. of Biomedical Engineering, Tongmyong University \\ ${ }^{2}$ Dept. of Computer Engineering, Tongmyong University, \\ ${ }^{3}$ Dept. of Media Engineering, Tongmyong University \\ sikang@tu.ac.kr',ghkim@tu.ac.kr² \\ Correspondence Author: asoh@tu.ac.kr ${ }^{3}$
}

\begin{abstract}
For the construction of biometric database from the source data which derived from personal portable device, HL7(Health Level Seven)and CDA(Clinical Document Architecture)standard should be used as application layer protocol. HL7 and CDA standard are protocols which would be used for the communication of medical information among hospital system, but it cannot be connected with mobile devices. Thus, for the u-healthcare monitoring service message conversion gateway should be utilized under the mobile environments. In this study, we designed message conversion gateway and diagnosis support system which control messages among ISO/IEEE 11073 and HL7 CDA. Using this system, medical team located on remote site can manage real time based biometric data.
\end{abstract}

Keywords: HL7, ISO/IEEE 11073, PHD, U-healthcare, Medical Information

\section{Introduction}

Nowadays the requirement for the welfare is increasing rapidly as life level of people is growing up year by year. Requirement of people who want to utilize IT technology depending on their high life quality is increasing continuously. Thus, home healthcare service requirement is also increasing for the preventive healthcare and wellness. For this uhealthcare service system communication between transmission system which transmit measured biometric data and interface system is very important.

Smart healthcare service provides preventive healthcare management and wellness based on smart phone. However, medical healthcare service can handle only first aid kit as yet, thus many kinds of skills are rising these days. Especially, healthcare service using smart phone is suggested and it manages medical treatment, wellness, safety and so on.

In this study, we try to design diagnosis support system in addition to the message conversion gateway. That gateway should assure the connection among home healthcare medical devices under the IEEE 11073 PHD (Personal Health Device)and HL7(Health Level Seven) CDA(Clinical Document Architecture)environments.

\section{Related Works}

User's biometric data transmission and HL7 environment is important for the study of Diagnosis support system. ISO/IEEE 11073 PHD standard is also important for the gateway. 


\subsection{ISO/IEEE11073 PHD}

ISO/IEEE11073 20601 and 11073 104xx standard have objective for the optimized communication among personnel healthcare devices. Standard documents provide public and independent standard to the u-health measurable and manageable devices.

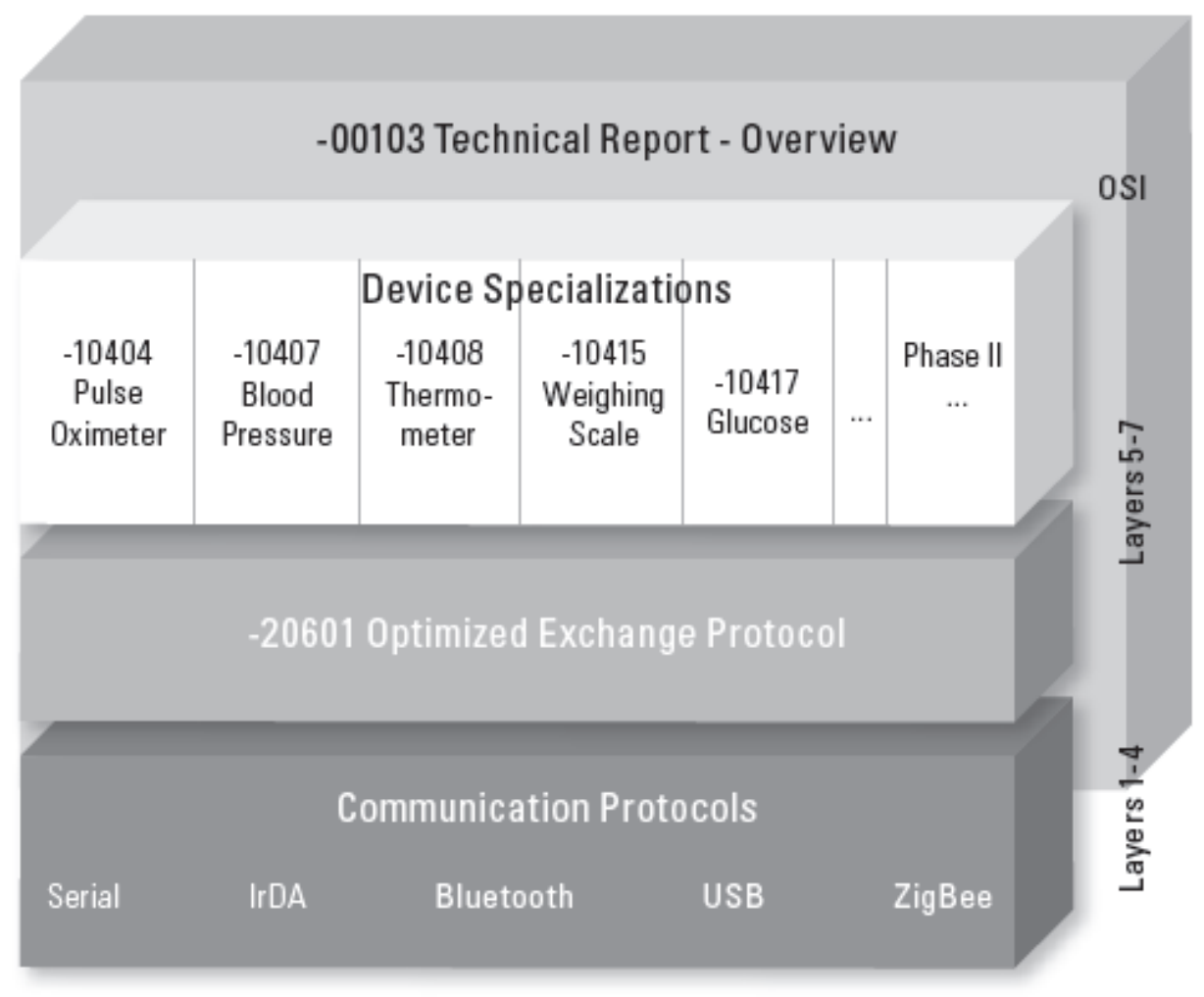

Figure 1. ISO/IEEE11073 PHD Structure

Figure 1 shows ISO/IEEE11073 PHD structure. At the bottom of the figure shows communication protocols. It represent transmission layer. Optimized IEEE 11073-20601 communication protocol is located upper communication protocols layer and it defines protocol for data exchange between measuring device and gateway.

Device Specializations layer located on top of this structure defines the standard of home healthcare devices. Existing healthcare system require gateway platform that support IEEE 11073-20601 application profile-optimized exchange protocol. It has many restrictions for providing u-healthcare service and healthcare devices including IEEE 11073 platform. Recently, Android 4.0 support Bluetooth HDP as support the connection of healthcare devices.

\subsection{Bluetooth HDP}

Bluetooth HDP is a application which is developed for the Bluetooth wireless technology targeting medical and healthcare devices. It defines measuring devices like blood pressure gauge, blood glucose monitoring device, scale, thermometer as source agent and defines other data collection devices like mobile phone, note book, desk top as sink (gateway).

As shown in Figure 2, ISO/IEEE 11073-104xx Device Specializations can be used with ISO/IEEE 11073-20601 Personal Health Data Exchange Protocol. Thus, every medical device 
which is defined by ISO/IEEE 11073 can be used on the Bluetooth HDP environment.

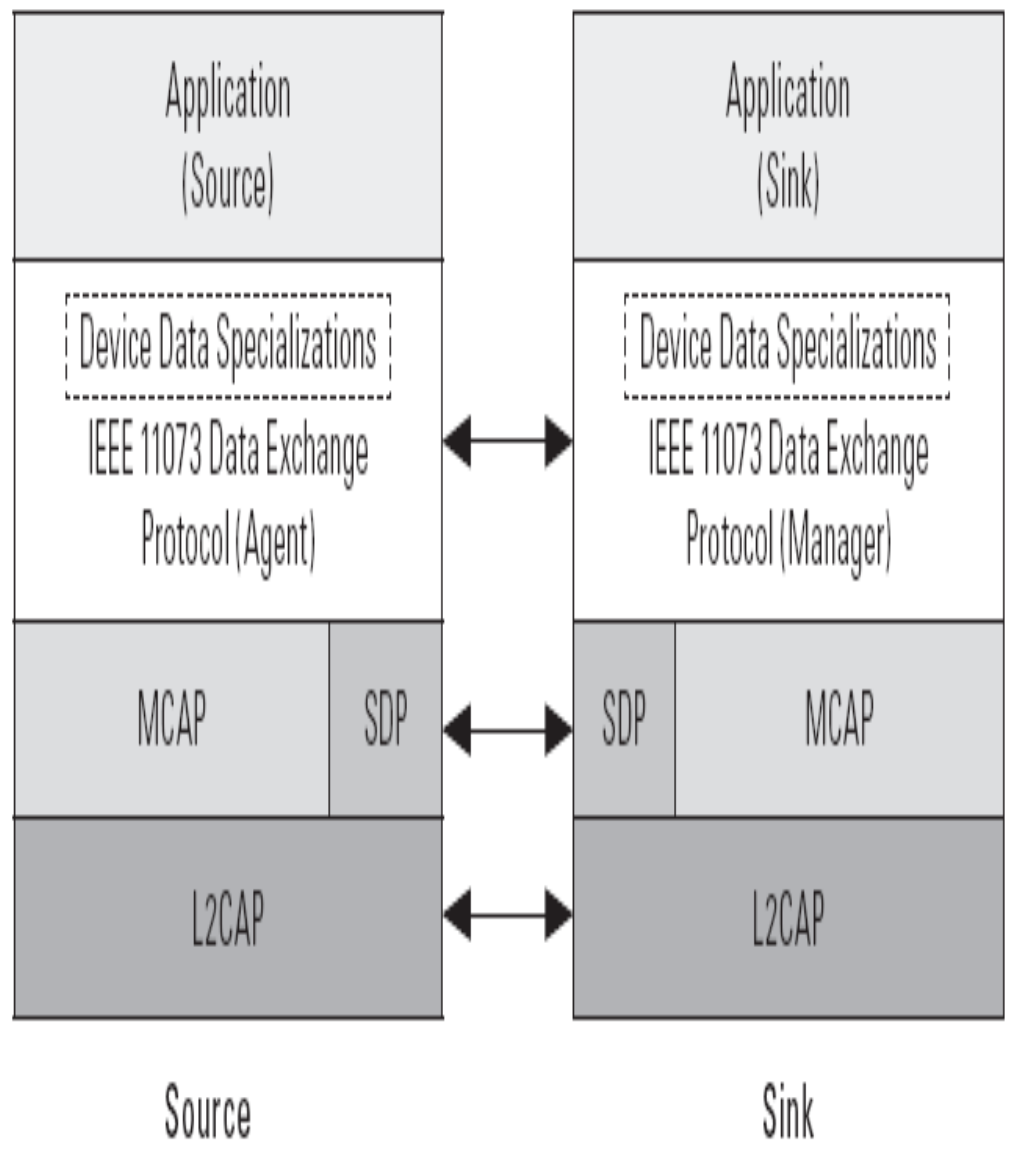

Figure 2. Bluetooth HDP Protocol Model

\subsection{Bluetooth HDP under IEEE 11073 Standards}

ISO/IEEE 11073 PHD standard is designed to communicate between u-health measuring device and u-health gateway. ISO/IEEE 11073-20601 and ISO/IEEE11073-104xx standard targeted the optimized communication among personal healthcare devices. Thus, if they use Bluetooth HDP, then they can use all of medical devices defined by ISO/IEEE 11073.

\subsection{Message Conversion between IEEE 11073 and HL7 CDA}

For the construction of PHR (Personal Health Record) database, from the source biometric data measured by mobile phone it should be followed by the guideline of HL7 CDA standard. As shown in Figure 3, although biometric data measured from personal healthcare device could be transmitted as standard IEEE 11073 PHD, it can't connect HL7 CDA protocol directly. Thus, there are needs conversion gateway between ISO/IEEE 11073 and HL7 CDA. 


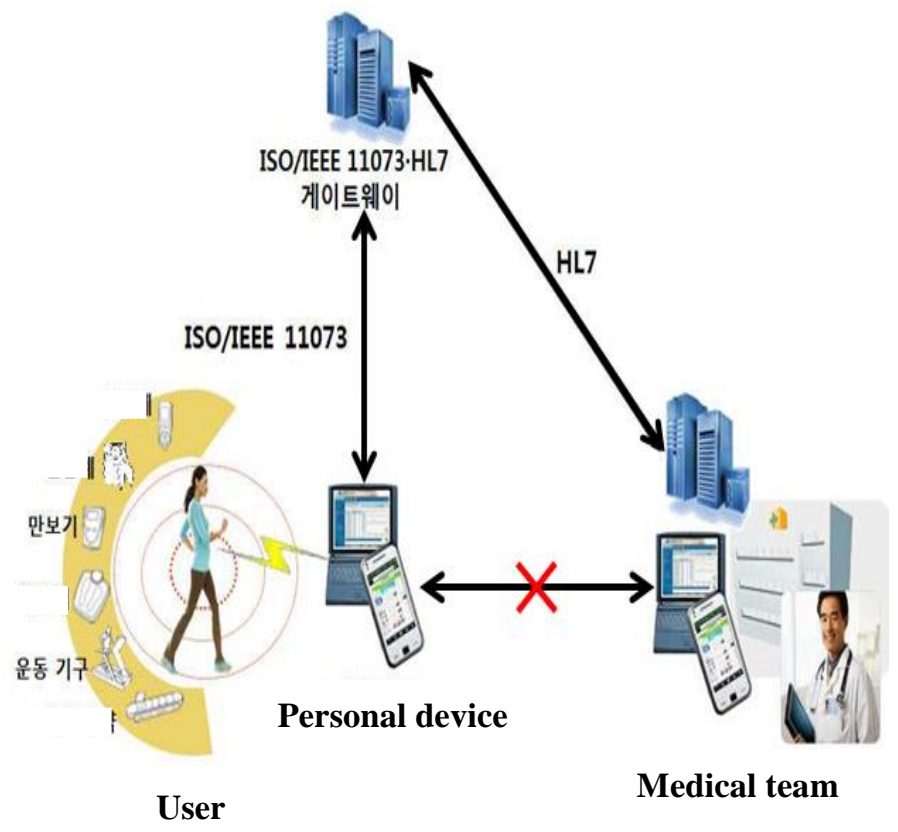

Figure 3. U-healthcare Service Structure

We try to design diagnosis support system utilizing HL7 conversion application. In this system, it receives biometric data from home healthcare device which is based on IEEE11073 PHD standard.

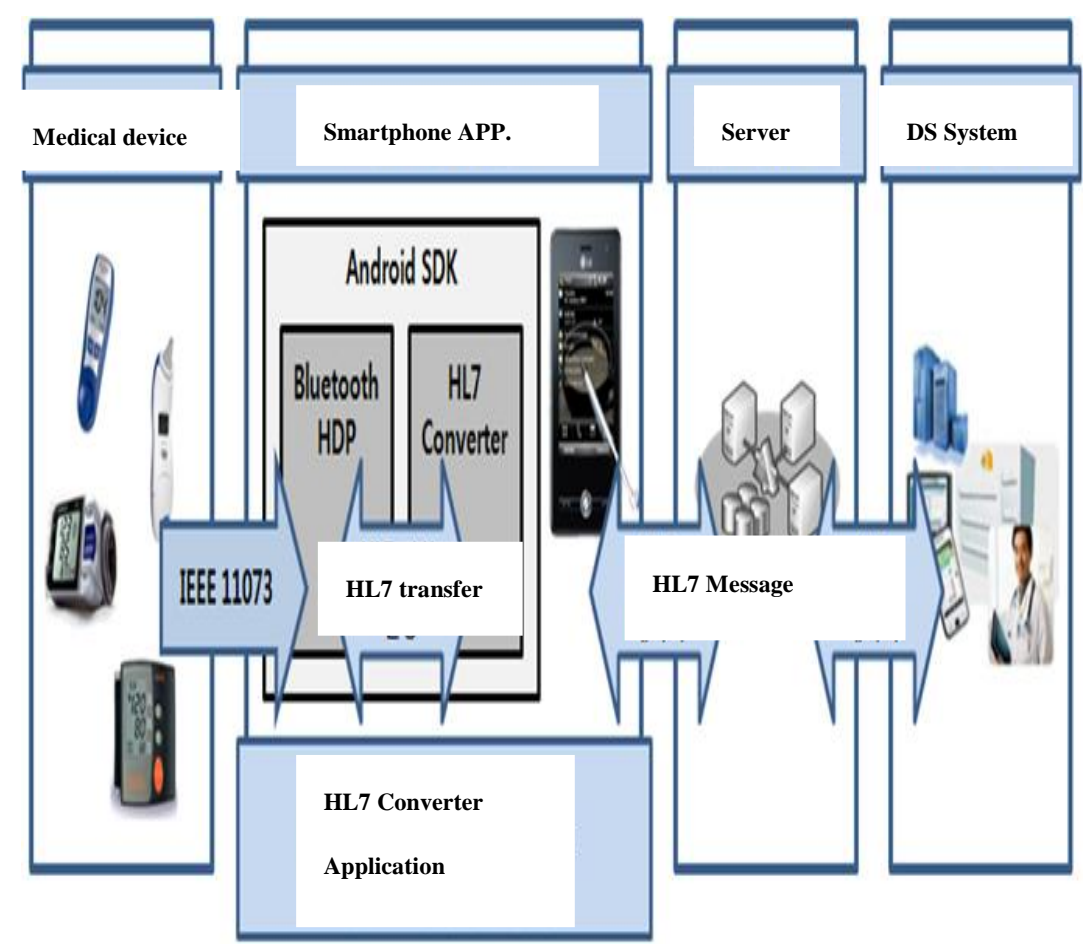

Figure 4. Information Transmission Structure of Diagnosis Support System 


\section{Design of Diagnosis Support System}

HL7 based diagnosis support system receives users biometric data thru Bluetooth HDP and transmits the converted messages to health server after changing it into HL7 v2x standard message. The structure of software module of HL7 is as shown in Figure 5.

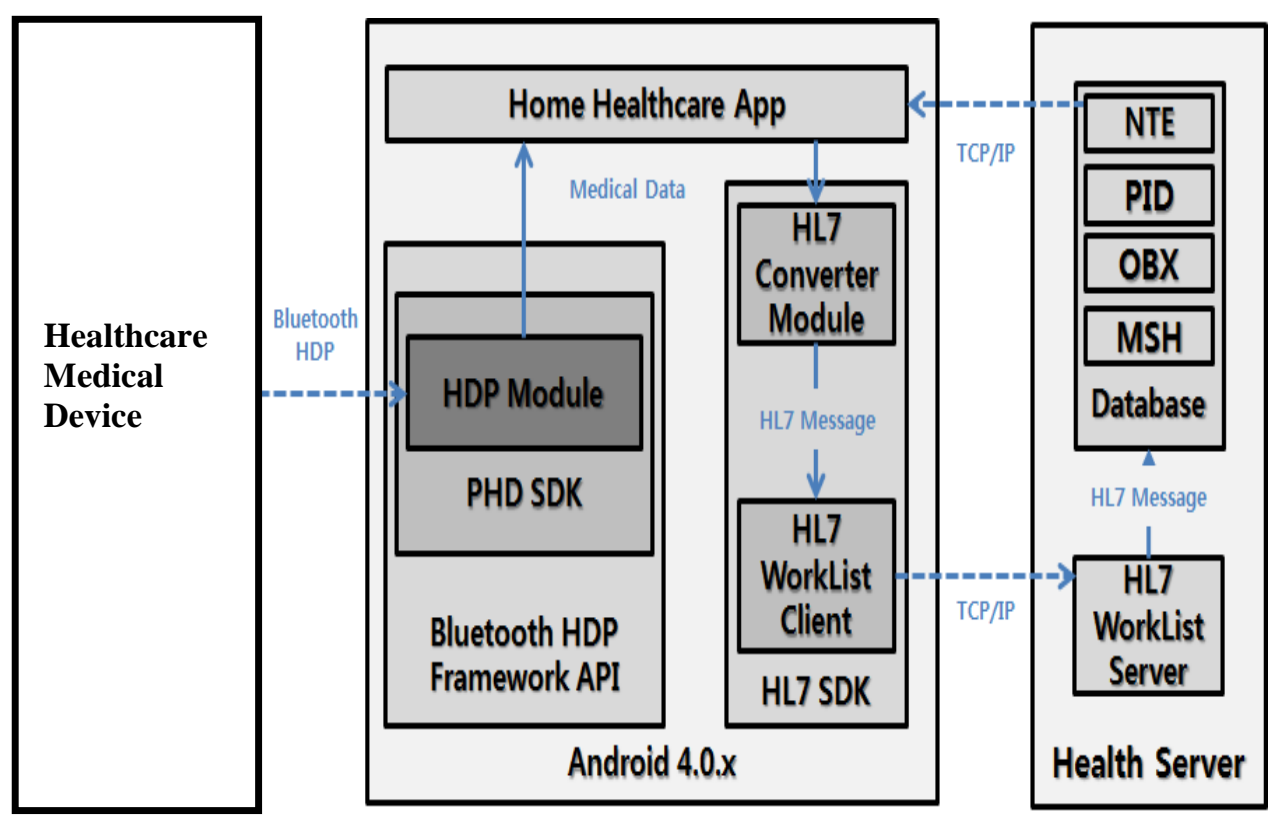

Figure 5. Configuration of HL7 Software Module

\subsection{Bluetooth HDP based Application}

Figure 6 shows configuration of smart phone application. The data from healthcare medical device is transmitted to the smart phone by Bluetooth HDP, and then would be stored or output user interface thru monitoring application.

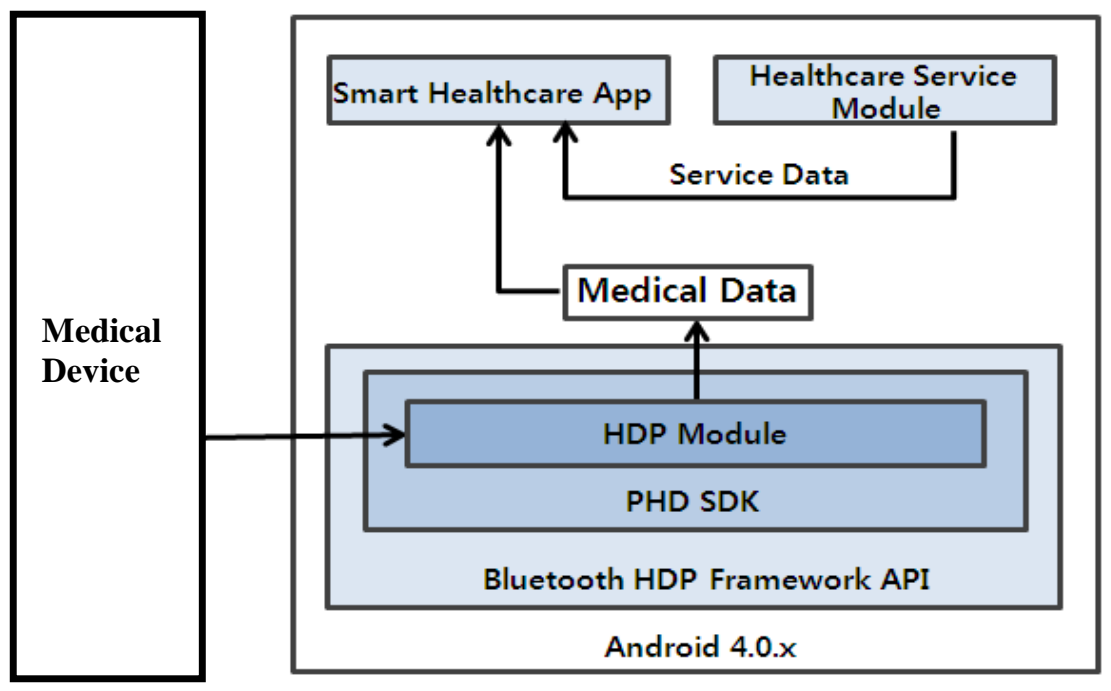

Figure 6. Configuration of Application System 


\subsection{IEEE 11073 Information Creation}

Figure 7 shows the procedure of IEEE11073 information creation. Service provider can monitor as comparing the created data with IEEE11073 PHD standard data.

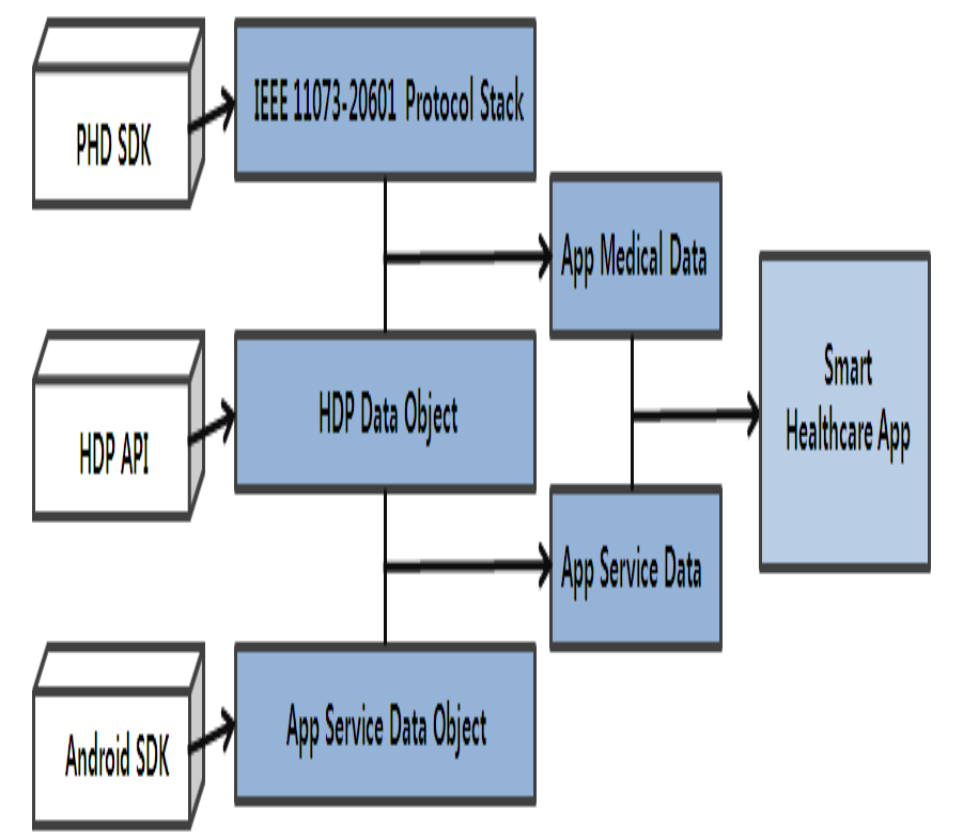

Figure 7. IEEE 11073 Information Creation Procedure

\subsection{HL7 Conversion Application}

HL7 conversion application is the message conversion gateway between ISO/IEEE 11073 and HL7 CDA. It receives biometric data via home healthcare medical devices and gathers the personal healthcare, device attribute data from user interface. After these step, it converts the message into HL7 after transmitting the data to the HL7 Converter Module.

After all, the converted HL7 messages would be sent to HL7 Worklist Server in Health Server. Thus, HL7 CDA based medical information could be transmitted as IEEE 11073 PHD standard biometric data is sent through TCP/IP.

\subsection{Health Server}

Health server does the function of the service provider and stores the message into database after confirming HL7 message received from HL7 Worklist server. It can support the diagnosis as managing biometric data received from user, and transmit to the user interface thru NTE (diagnosis information management).

\section{Conclusion}

In this study, we designed HL7 based diagnosis support system which can communicate efficient message and take remote medical treatment among personal devices and hospital systems. This diagnosis support system assures connection, security and reliability of measured biometric data between home healthcare device and gateway. It also gives simple and efficient remote treatment using conversion application which converts IEEE11073 PHD standard biometric data into HL7 message. In addition to these ones, it can give individual 
customized treatment service when the medical team located on remote site manages sensors as real time (data amount, data frequency etc.)

\section{References}

[1] C.S. Kim, "The Trends and Prospects of Health Information Standards: Standardization Analysis and Suggestions", Department of Radiological Science, College of Health Sciences, Catholic University of Pusan, (2008).

[2] B. Orguna and J. Vub, "HL7 ontology and mobile agents for interoperability in heterogeneous medical information systems", Computers in Biology and Medicine, vol. 36, (2006), pp. 817-836.

[3] T. R. Lee and M. K. Choi, "Development And Implementation of System for Delivery of Emergency Patient's Basic Information Between Related Hospitals", Journal of Health Science \& Medical Technology, vol. 29, no. 2, (2003), pp. 67-80.

[4] J. Escayola, M. Martnez-Espronceda and P. Muoz, "Seamless Integration of ISO/IEEE11073 Personal Health Devices and ISO”, TELEMEDICINE JOURNAL AND E HEALTH, vol. 16, no. 10, (2010).

[5] S. Carot-Nemesio and J. A. Santos-Cadenas, "OPENHEALTH - The Open Health FLOSS Implementation of the ISO/IEEE 11073-20601 Standard”, HEALTHINF - PROCEEDINGS, vol. 3, no. 1, (2010).

\section{Authors}

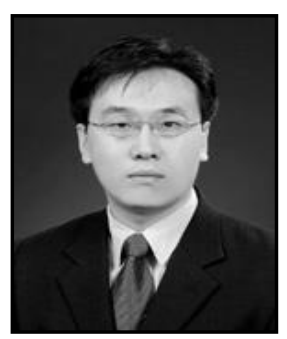

Sung-In Kang, received the Ph.D. degrees in electrical communication from Korea Maritime University in 2004. He is currently a Full-Time Instructor in Dept. of Computer Engineering, TongMyong University. His research interests are wireless sensor network, signal processing, and artificial intelligence

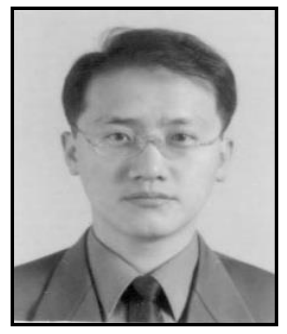

Gwan-Hyung Kim, received the Ph.D. degrees in electrical communication from Korea Maritime University in 2002. He is currently a Full-Time Instructor in Dept. of Computer Engineering, TongMyong University. His research interests are intelligent control, signal processing, and robotics.

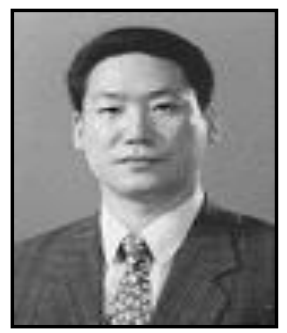

Am-Suk Oh, received the B.S. and M.S. degrees in computer science from Pusan National University and Chung-ang University, respectively. He received $\mathrm{Ph}$.D degree at the computer engineering of Pusan National University. He is currently with the Department of Multimedia Engineering, TongMyong University of Information Technology as Professor. 
International Journal of Bio-Science and Bio-Technology Vol.5, No.6 (2013) 